\title{
Comparison of Five Liver Fibrosis Indexes with Serum Levels of Laminin and N Terminal Peptide of Procollagen Type III in Chronic Hepatitis Patients
}

\author{
Hadi Parsian ${ }^{1,2}$, Mohammad Nouri ${ }^{3}$, Ali Rahimipour ${ }^{4}$, \\ Mohammad Hossein Somi ${ }^{3}$ and Durdi Qujeq ${ }^{1,2}$ \\ ${ }^{1}$ Department of Biochemistry and Biophysics, \\ Babol University of Medical Sciences, Babol, \\ ${ }^{2}$ Cellular and Molecular Biology Research Centre, \\ Babol University of Medical Sciences, Babol, \\ ${ }^{3}$ Liver and Gastrointestinal Diseases Research Centre, \\ Tabriz University of Medical Sciences, Tabriz, \\ ${ }^{4}$ Department of Clinical Biochemistry, \\ Shahid Beheshti University of Medical Sciences, Tehran,
}

Iran

\section{Introduction}

Different types of disease for example viral hepatitis, ethanol and biliary tract disease can produce liver fibrosis (Stalnikowitz \& Weissbrod AB, 2003). Liver fibrosis can be divided into those diseases in which the fibrosis is portal based and those that are central based. Chronic viral hepatitis (viral and autoimmune), chronic cholestatic diseases and hemochromatosis are the major portal based diseases and steatohepatitis and chronic venous outflow obstruction of any cause can causes central based diseases (Benyon \& Arthur, 2001). Chronic viral hepatitis (especially hepatitis B and C) and autoimmune hepatitis are the major causes of liver fibrosis, especially in developing countries.

The hepatitis B virus (HBV) belongs to the Hepadnaviridae family (Marco et al, 1999) and it is estimated worldwide HBV infection prevalence is to be around 350 million chronic carriers (Kao \& Chen, 2002). Infection with the hepatitis C virus (HCV) in the general population varies from $0.2 \%$ to $18 \%$ (Neighbors, 2007). HCV can infect liver cells and is able to cause severe inflammation of the liver with serious complications. Autoimmune hepatitis $(\mathrm{AIH})$ similar to the above mentioned diseases, is a chronic disease that is associated with some autoantibodies such as antinuclear antibodies (ANA), liver-kidney microsomal antibodies (LKM), anti mithochondial antibody (AMA), soluble liver antigen (SLA), smooth muscle antibodies (SMA) and hypergammaglobulinemia. The prevalence of this disesesis is between 0.1 and 1.2 cases per 100,000 in Western Europe and North America but only 0.080.015 cases per 100,000 in Japan (Manna \& Strassburg, 2001). 


\subsection{Hepatic fibrosis}

In response to liver injury, because of any cause, accumulation of extracellular matrix (ECM) proteins (collagen types, proteoglycans, fibronectin and laminin) will occur. The ECM scaffold is taken down by some special matrix proteinases while activated stellate cells (SC) undergo apoptosis and try to restore the normal tissue structure. Hepatic SC will produce matrix metalloproteinases (MMPs) to regulate the extracellular degradation of matrix proteins. Ultimately, as a result of an imbalance between fibrogenesis and fibrolysis, collagen deposition will occurs and scar will form. When scarring progressed, architectural distortion, liver fibrosis and ultimately cirrhosis will occur (Stalnikowitz \& Weissbrod, 2003). Figure 1 illustrates this phenomenon.

\subsection{Hepatic inflammation}

After liver cell injury, recruitment of leukocytes will takes place (Marra, 1999). Leukocytes together with kupffer cells will produce compounds that modulate stellate cell behavior. Nitric oxide (NO) and inflammatory cytokines, such as tumor necrosis factor a (with stimulatory ability on the stellate cell for collagen synthesis) will produce by monocytes and macrophages. In addition, kupffer cells can stimulate matrix synthesis by stellate cells through the actions of transforming growth factor $\beta$ (TGF- $\beta$ ) and reactive oxygen species (ROS) (Stalnikowitz \& Weissbrod, 2003; Granger \& Kubes, 1994) -Figure 1.

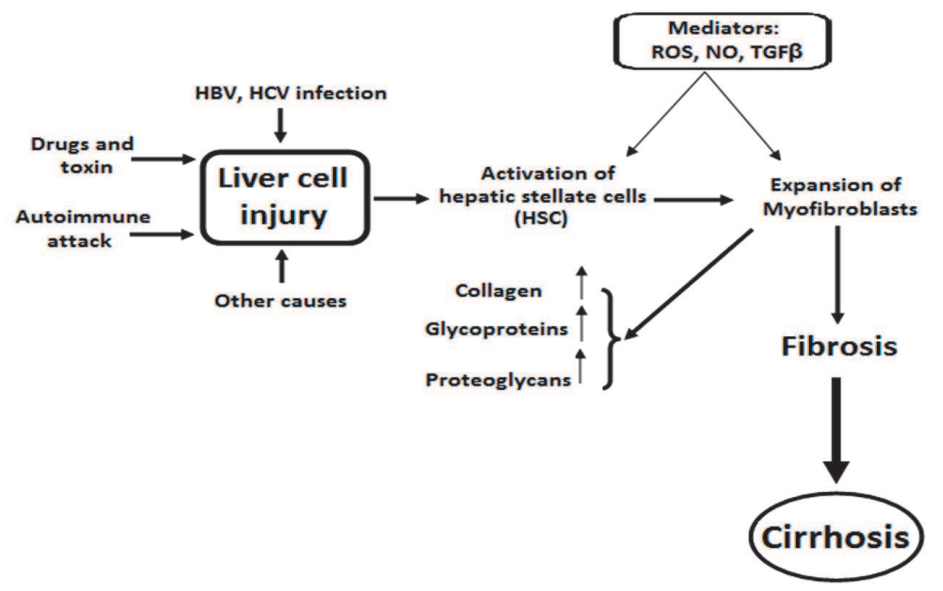

Fig. 1. Proposed mechanism of activation of hepatic stellate cells leading to fibrosis and cirrhosis. ROS, reactive oxygen species; NO, nitric oxide; TGF $\beta$, transforming growth factor $\beta$ (resulted from Gressner et al., 2007).

\subsection{Assessment of severity of liver fibrosis}

Now, the gold standard for assessment of liver fibrosis is liver biopsy. For scoring liver fibrosis, several systems have been proposed that all of them are based on visual assessment of collagen staining of liver biopsy samples. These are including the histology activity index (HAI), the modified Knodell score, the Ishak score and the Metavir score (Marcellin et al, 2002). Recently a newly scoring system is proposed and named modified Knodell score system. The grading of liver inflammation injuries was scored from 0 to 18. The staging of liver fibrosis was scored from 0 to 6 and is according to the Table 1 (Knodell et al., 1981; Ishak et al., 1995). 


\begin{tabular}{|c|c|}
\hline Score & Fibrosis stage \\
\hline 0 & No fibrosis \\
\hline 1 & Fibrous expansion of some portal areas, with or without short fibrous septa \\
\hline 2 & Fibrous expansion of most portal areas, with or without short fibrous septa \\
\hline 3 & $\begin{array}{l}\text { Fibrous expansion of most portal areas with occasional portal to portal (P-P) } \\
\text { bridging }\end{array}$ \\
\hline 4 & $\begin{array}{l}\text { Fibrous expansion of portal areas with marked bridging [portal to portal (P-P) } \\
\text { as well as portal to central }(\mathrm{P}-\mathrm{C})]\end{array}$ \\
\hline 5 & $\begin{array}{l}\text { Marked bridging (P-P and/or P-C) with occasional nodules (incomplete } \\
\text { cirrhosis) }\end{array}$ \\
\hline 6 & Cirrhosis, probable or definite \\
\hline
\end{tabular}

Table 1. Modified Knodell score system for liver fibrosis staging (Knodell et al., 1981).

Liver biopsy, as a clinical tool, has some major limitations (Rossi et al., 2007). Most famous limitations of liver biopsy are: fibrosis staging systems (with this assumption that there is a linear increase in the severity of fibrosis between stages) (Rosenberg et al., 2004), sampling error (a 10-15 mg sample of tissue represents a tiny fraction of an organ weighing $1500 \mathrm{~g}$ ) (Regev et al., 2002) and inter-observer variation amongst pathologists in categorizing the degree of fibrosis (Westin et al., 1999).

For assessment of liver fibrosis, in addition to invasive method (liver biopsy), there are some serum-based noninvasive markers individually or in an algorithm-based score. Noninvasive markers in assessment of liver necroinflammatory injuries are the best markers, because they can analyze frequently during the treatment protocol of liver fibrosis and also for highlighting the efficacy of treatment.

The most famous individual markers for assessment of liver fibrosis are: liver function tests (aspartate aminotransferase: AST and alanine aminotransferase: ALT) that reflect hepatocyte damage, bilirubin and alkaline phosphatase that show biliary obstruction and albumin and prothrombin time (PT) that reveal biosynthetic function of liver. These tests only provide information about important aspects of liver function but they do not assess severity of liver fibrosis or cirrhosis (Rossi et al., 2007). Other serum markers such as a-2-Macroglobulin (Naveau et al., 1994), apolipoprotein A1 (Poynard et al., 1991), haptoglobin (Bismut et al., 2001), matrix metalloproteinases (MMPs) and their tissue inhibitors (TIMPs) (Leroy et al., 2004), hyaluronic acid, collagen markers, aminoterminal propeptide of type III procollagen (PIINP) and laminin (LN) are proposed, too, as surrogate indices instead of liver biopsy (Gressner et al., 2007). New researches indicated that these individuals' serum markers have limited accuracy in predicting hepatic fibrosis and proposed that the individual markers are useful for establishing the presence, but not absence, of fibrosis. Due to the limitations of individual markers to assess liver fibrosis, algorithms or indices combining the results of panels of markers have been studied which reportedly improve diagnostic accuracy. The serum marker panels have been proposed as an alternative to liver biopsy (Crockett et al., 2006; Castera \& Pawlotsky, 2005). 
Some of the available serum marker panels include HCV FibroSURE, ASH FibroSURE, NASH FibroSURE, FIBROSpect II, Hepascore, Forns score, APRI, AAR, AP, SHASTA, Fibro $\mathrm{Q}$ and FIB4 index and European Liver Fibrosis Group (ELFG) algorithm. Some are beyond the scope of this chapter; however five of them that evaluated in this study, briefly are discussed here. In this study the serum levels of LN and PIIINP, in addition to the five serum marker panels of liver necroinflammatory injury and the histologicall evaluation of liver biopsies according to the modified Knodell score system were studied.

\subsubsection{Laminin (LN)}

One of the main glycoproteins of the basement membrane is laminin (LN). This molecule participates in a series of biological events such as the maintenance of the cytoskeleton (Rosa \& Parise, 2008). Hpatocytes and sinusoidal cells are able to synthesis of LN (Rosa \& Parise, 2008; Gressner et al., 2007). Deposition of LN and collagen determine the formation of a true basement membrane along sinusoids in the liver. Increased in production of LN in the liver and a lack of degradation of this glycoprotein by liver endothelial cells, are the major causes of elevated serum levels of this protein in fibrotic and cirrhotic liver. It is proposed that laminin serum concentration is a sensitive screening test for hepatic fibrotic disease (Rosa \& Parise, 2008).

\subsubsection{Aminoterminal propeptide of type III procollagen: PIIINP}

During the processing of type III procollagen, the PIIINP molecule is produced by a specific $\mathrm{N}$-proteinase. There are evidences that proposed purified preparations of the PIIINP molecule will degrade by scavenger receptors located on the liver endothelial cells. In various clinical situations such as liver fibrosis, metabolism of type III collagen will alter, therefore measurement of PIIINP concentration in serum will has some clinical application. Some studies revealed that there is a significantly increased serum level of PIIINP in patients with liver fibrosis and cirrhosis. It proposed that these increased serum levels are related to increased hepatic synthesis of connective tissue components and also a decreased in hepatic clearance ability (Smedsr et al., 1990).

\subsubsection{Serum marker panels/models}

\subsubsection{Age-platelet (AP) index}

$\mathrm{AP}$ index is a simple index that derived from age and platelet counts and is calculate as follow:

$$
\begin{gathered}
\text { Age (years) }<30=0 ; 30-39=1 ; 40-49=2 ; 50-59=3 ; 60-69=4 ; \geq 70=5, \\
\text { Platelets counts }(109 / 1): \geq 225=0 ; 200-224=1 ; 175-199=2 ; \\
150-174=3 ; 125-149=4 ;<125=5
\end{gathered}
$$

The sum of age and platelet counts scores according to the above mentioned scoring system, named AP index (Poynard et al,. 1997).

\subsubsection{AST to platelet ratio index (APRI)}

Several studies have suggested that the APRI may be a useful noninvasive marker of hepatic fibrosis in patients with chronic liver disease. APRI Score was initially described by Wai et al (Wai et al., 2003) and calculated by this formula: 


$$
\text { APRI } \left.=\left((\text { AST } / \text { upper limit of normal }) / \text { platelet count }\left(10^{9} / \mathrm{L}\right)\right) \times 100\right)
$$

\subsubsection{AAR index}

Another simple index that used for evaluation of liver fibrosis is AAR index. One can calculate this index by dividing the levels of AST to ALT (Williams et al., 1988).

\subsubsection{Fibro $Q$}

Fibro Q is another index that dependent to age (years), AST level, platelet count and also PT International Normalized Ratio (INR) and can be calculating by the following formula (Hsieh et al., 2009).

$$
\text { Fibro } \mathrm{Q}=[(10 \times \text { age }(\text { years }) \times \text { AST } \times \text { PT INR }) /(P L T \times \text { ALT })] .
$$

\subsubsection{FIB4}

FIB-4 is an index that is dependent to age (years), AST and ALT levels (U/L) and platelet count (Sterling et al., 2006). It can be calculated simply by the following formula.

$$
\text { FIB-4 } \left.=[\text { age }(\text { year }) \times \text { AST }(\mathrm{U} / \mathrm{L})] /\left[\operatorname{PLT}\left(10^{9} / \mathrm{L}\right) \times \operatorname{ALT}(\mathrm{U} / \mathrm{L})\right)^{1 / 2}\right) .
$$

The aim of this study was identify the efficiency of the extracellular matrix components i.e. LN and PIIINP in comparison with five noninvasive fibrosis models (AP, APRI, AAR, Fibro $\mathrm{Q}$ and FIB4 index) and to recognize the most important model for discrimination of patients with liver fibrosis (chronic hepatitis B, C and auto immune hepatitis (AIH) patients) vs. control and also for discrimination of patients with severe liver fibrosis (stage $\geq 3$ ) vs. mild liver fibrosis (stage $\geq 2$ ).

\section{Methods}

\subsection{Study population}

Sixty-two patients; ( 35 men and 27 women, mean \pm SD: $35.4 \pm 11.3$ years) were enrolled in this study. Among these patients 35 patients had hepatitis B, 14 had hepatitis $C$ and 13 were autoimmune hepatitis (AIH). The patients were selected from the persons who referred to Liver and Gastrointestinal Disease Research Center of Tabriz, Babol and Gorgan (Gonbad) University of Medical Sciences for diagnosis of the disease.

Control sera for the determination of LN and PIIINP were obtained from 20 healthy volunteer's person who referred to the Tabriz University of Medical Sciences (10 women and 10 men, mean \pm SD: $42 \pm 14.7$ years). Control groups had normal serum levels of aminotransferases and alkaline phospahtase. None of the controls had a history of gastrointestinal bleeding and chronic liver disease, smoking (never smoker), alcohol intake (never drinker), a family history of hepatitis and liver disease, active intravenous drug abuse and a liver transplantation, according to the information's gathered in the questionnaire form. Persons who smoked ( $>1$ cigarette/day) and drunk alcohol ( $>1$ gr/day), were classified as smoker and alcohol drinker.

All of the subjects were informed about the study and their consents were taken. This study was approved by Tabriz Medical University Ethical Committee.

\subsubsection{Inclusion criteria}

Patients were included in the study, if they were positive for serum hepatitis B surface antigen (HBs-Ag) or $\mathrm{HCV}$ antibodies, and having persistently elevated serum 
aminotransferases greater than 1.5 times the upper limit of the reference range for at least six months. AIH patients were diagnosed according to the International Autoimmune Hepatitis Group Report protocol (Manna \& Strasburg, 2001). For assessment of liver fibrosis scores, all patients underwent liver biopsy as part of the normal diagnostic procedure and were subsequently sub-classified according to the score for the histological activity index (HAI).

\subsubsection{Exclusion criteria}

Patients with a history of gastrointestinal bleeding and other chronic liver disease (such as Wilson's disease, hemochromatosis, alpha1-antitrypsin deficiency, biliary disease, hepatocellular carcinoma), active intravenous drug abuse, and liver transplantation were excluded.

\subsection{Blood collection and sample preparation}

Blood samples $(7 \mathrm{ml})$ were collected after an overnight fast. After analyzing cell blood counts (CBC) on aliquots of whole blood, on remainder the samples, serum was separated (at 2500 $\mathrm{g}$ for 5 minutes) within one hour of blood collection. Standard LFT, including AST and ALT and hepatitis serology were performed on aliquots of each sample and recorded. Routine LFT and hepatitis serology was assayed using commercial available kits. Briefly AST and ALT were measured by colorimetric test (Ziestchem kit, Iran) with 2, 4 DNPH $(2,4$ dinitrophenylhydrazone) with Apel spectrophotometer (PD303S, Japan).

The hepatitis serology markers were analyzed by an ELISA reader (Norahan Fajr, Iran) and the following Kits. The AIH serology markers that analyzed were: antinuclear antibody (ANA; Biazyme ELISA kit, Birmingham, UK) and anti-LKM-1 (LKM-1: type 1 liver and kidney microsomes, Euroimmun ELISA kit, Germany), hepatitis B serology markers were: hepatitis B surface antigen (HBsAg; Diakey, shinjin Medics Inc. ELISA kit, Korea), hepatitis B Surface Antibody (HBsAb; Diakey, shinjin Medics Inc. ELISA kit, Korea) and hepatitis B Core Antibody (HBcAb; Dia-Pro ELISA kit, Italy) and hepatitis $C$ serology marker was: HCV antibody (HCVAb; Diakey, shinjin Medics Inc. ELISA kit, Korea). HCV RNA was assayed using a PCR kit (Roche Diagnostics, Mannheim, Germany) by an Eppendorf Thermocycler (Mastercycler Personal) according to the manufacturer's instructions. Two primers (Sinagene, Tehran, Iran) with the following sequences were used: first primer sequence 5'-GCA GAA AGC GTC TAG CCA TGG CGT-3' and second primer sequence 5'CTC GCA AGC ACC CTA TCA GGC AGT-3'.

The rest of the blood samples were stored at $-20^{\circ} \mathrm{C}$. The controls were dealt with in the same manner.

\subsection{Determination of serum LN and PIIINP}

\subsubsection{LN assay}

Serum LN concentrations were assayed using a LN EIA Kit (Takara Bio, product number: MK107) on an ELISA reader (BDSL, Immunoscan, Lab System, Switzerland) and the amount of LN was determined by a standard curve. The intra assay and inter-assay variability $(\mathrm{CV})$ of the procedure according to the manufacturer, were $4.0-5.7 \%$ and $0.3-5.0 \%$, respectively.

\subsubsection{PIIINP assay}

Serum PIIINP levels were assayed by a PIIINP ELISA Kit (USCN life and technology company, product no: E0573h) on an ELISA reader (BDSL, Immunoscan, Lab System, 
Switzerland). Briefly, a monoclonal antibody specific for PIIINP had been precoated onto a microplate. Standards and samples were pipetted into the wells and any PIIINP present was bound by the immobilized antibody. An enzyme-linked polycolonal antibody specific for PIIINP was added to the wells. Following a wash to remove any unbound antibody-enzyme reagent, a substrate solution was added to the wells and color developed in proportion to the amount of PIIINP bound in the initial steps. The color development was stopped and the intensity of the color was measured and ultimately the amount of PIIINP was determined by a standard curve. The intra assay variability $(\mathrm{CV})$ of the procedure according to the manusfacturer declares was $4-5-5.0 \%$.

\subsection{Histological assessment of liver damage}

Sixty-two patients underwent a percutaneous liver biopsy with a Trucut needle number 16 guided by B type ultrasound for assessing the presence and severity of liver disease. The biopsy fragments were fixed in a 10\% formalin solution for 12 hours and embedded in paraffin. Sections were stained with hematoxylin-eosin, Masson's trichrome and reticulin stain. Specimens were graded and staged according to the modified Knodell scoring system (Knodell et al., 1981) by a pathologist who was blinded to the results of serum indices in the study subjects. The grading system was scored from 0 to 18 and was based on sum of four indices:

1. Periportal or periseptal interface hepatitis (piecemeal necrosis, score 0-4)

2. Confluent necrosis (score 0-6)

3. Focal (spotty) lytic necrosis, apoptosis, and focal inflammation (score 0-4)

4. Portal inflammation (score $0-4$ ).

The fibrosis score were determined on the basis of the table 1 .

\subsection{Statistical analysis}

The SPSS 12.0 statistical package (SPSS for Windows 12.0, SPSS, Chicago, IL, USA) was used on statistical analysis. Data were expressed as mean $\pm \mathrm{SD}$, and P less than 0.05 was considered statistically significant. The mean patient serum LN and PIIINP levels between different types of chronic hepatitis and healthy controls were compared with analysis of variance (ANOVA). Five serum markers panels were calculated as mentioned in the introduction section 1.3.3. To assess the diagnostic accuracy of LN and PIIINP and also five serum marker panels for discrimination chronic hepatitis patients with severe liver fibrosis from healthy individuals, and also discrimination of patients with severe liver fibrosis vs. mild liver fibrosis, we plotted the receiver operating characteristic curve (ROC). Receiver operating characteristic curves were generated by plotting the relationship of the true positivity (sensitivity) and the false positivity (1- specificity) at various cut-off points of the tests. An AUC of 1.0 is characteristic of an ideal test, whereas 0.5 indicates a test of no diagnostic value (Zweig \& Campbell, 1993). Taking sensitivity and specificity into account, the cut-off points were selected according to max number of sensitivity add specificity. The diagnostic accuracy, sensitivity, specificity, positive predictive values (PPV) and negative predictive values (NPV) also were calculated.

\section{Results}

Histological examination of liver for fibrosis scoring revealed that 10, 19, 11, 9, 8,4 and 1 patient were at stages $0,1,2,3,4,5$ and 6 of liver fibrosis, respectively. In the other hand, 
$35 \%$ of patients to be suffering from significant fibrosis (stage $\geq 3$ ). In addition $32.2 \%$ of patients had liver inflammation grades $\geq 5$. Hepatitis serology revealed that $56.4 \%$ of the patients were suffering from chronic hepatitis B, 22.5\% from chronic hepatitis C and $20.9 \%$ from autoimmune hepatitis. In table 2 characteristics of chronic hepatitis patients and controls are shown. The results of laboratory tests showed that the serum levels of AST and ALT in patients groups were significantly higher than in the control group $(p<0.001)$ but the differences in platelet count and prothrombin time were not significant.

The results also showed increased serum LN and PIIINP levels in patients, as compared with the healthy controls $(\mathrm{p}<0.001)$.

\begin{tabular}{|c|c|c|c|}
\hline Variables & Patients $(\mathrm{n}=62)$ & Controls $(\mathrm{n}=20)$ & P value \\
\hline Male/Female $(\mathrm{n})$ & $35 / 27$ & $10 / 10$ & - \\
\hline Age in years & $35.4 \pm 11.3$ & $42.0 \pm 14.7$ & - \\
\hline Platelet count $\left(10^{9} / \mathrm{L}\right)$ & $225.7 \pm 122.4$ & $179.7 \pm 25.5$ & 0.051 \\
\hline Prothrombin time (s) & $13.56 \pm 1.65$ & $13.15 \pm 1.47$ & 0.345 \\
\hline ALT, IU/L & $143.0 \pm 145.6$ & $27.3 \pm 6.4$ & $<0.001$ \\
\hline AST, IU/L & $101.1 \pm 143.1$ & $28.3 \pm 6.5$ & $<0.001$ \\
\hline Laminin, $\mathrm{ng} / \mathrm{ml}$ & $91.9 \pm 20.9$ & $46.1 \pm 10.1$ & $<0.001$ \\
\hline PIIINP, $\mathrm{ng} / \mathrm{ml}$ & $6.8 \pm 1.7$ & $4.5 \pm 1.1$ & $<0.001$ \\
\hline
\end{tabular}

Table 2. Characteristics of the patient and control groups included in the study.

The mean serum LN and PIIINP concentrations in patients with HBV, HCV, AIH and healthy controls are presented in Table 3 . These results showed statistically increased serum LN and PIIINP levels in patients as compared with healthy controls $(\mathrm{P}<0.001)$.

The patient's serum LN and PIIINP levels in different stages of liver fibrosis are presented in Table 4. Almost in all stages of hepatic fibrosis, the serum levels of LN and PIIINP, were higher than those of the healthy group $(\mathrm{p}<0.05)$. In stage 0 of liver fibrosis the LN and PIIINP serum concentrations did not differ compare from the healthy controls. In fibrosis stage 6, which was represented by only one patient, the serum levels of LN and PIIINP are presented.

\begin{tabular}{|c|c|c|}
\hline Disease & LN & PIIINP \\
\hline HBV & $92.0 \pm 20.1^{*}$ & $6.6 \pm 1.9^{*}$ \\
\hline HCV & $92.8 \pm 24.1^{*}$ & $7.4 \pm 1.5^{*}$ \\
\hline AIH & $90.6 \pm 18.4^{*}$ & $6.8 \pm 1.4^{*}$ \\
\hline Total & $91.9 \pm 20.9^{*}$ & $6.8 \pm 1.7^{*}$ \\
\hline Healthy controls & $46.1 \pm 10.1$ & $4.5 \pm 1.1$ \\
\hline
\end{tabular}

* $P$ value $\leq 0.001$

Table 3. Comparison of LN and PIIINP serum concentration in different types of chronic hepatitis and control group. Data are presented as Mean $\pm \mathrm{SD}(\mathrm{ng} / \mathrm{ml})$. 
Figure 2 ( $a$ and b), are illustrated scatter plots for serum LN and PIIINP level $(\mathrm{ng} / \mathrm{ml})$ in hepatitis patients in various grades of inflammation. According to these figures, patients in higher grades of liver inflammation had higher serum concentrations of LN and PIIINP and the highest level were observed in grades of 5-12 of liver inflammation. We had no patient in inflammation grades of 13-18.

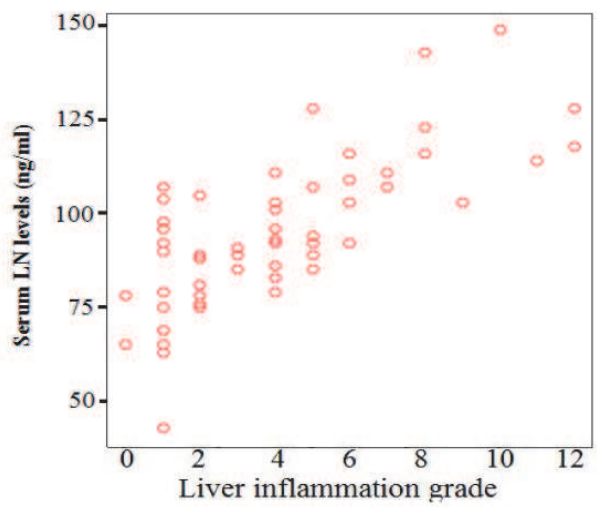

(a)

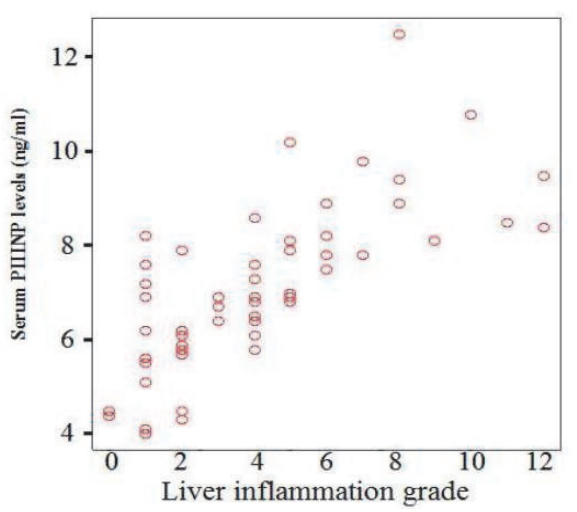

(b)

Fig. 2. Scatter plots for serum LN (a) and PIIINP (b) level $(\mathrm{ng} / \mathrm{ml})$ in patients at various grades of inflammation.

\begin{tabular}{|c|c|c|}
\hline Stage & LN & PIINP \\
\hline 0 & $63.0 \pm 12.1$ & $4.5 \pm 0.5$ \\
\hline 1 & $85.7 \pm 6.3^{*}$ & $6.1 \pm 0.9^{*}$ \\
\hline 2 & $94.0 \pm 10.2^{* *}$ & $7.0 \pm 0.8^{*}$ \\
\hline 3 & $100.6 \pm 8.6^{* *}$ & $7.7 \pm 0.6^{* *}$ \\
\hline 4 & $104.2 \pm 20.2^{* *}$ & $8.0 \pm 1.5^{* *}$ \\
\hline 5 & $130.2 \pm 13.7^{* *}$ & $9.8 \pm 0.8^{* *}$ \\
\hline $6 \dagger$ & 143.0 & 12.5 \\
\hline Total & $91.9 \pm 20.9^{* *}$ & $6.8 \pm 1.7^{* *}$ \\
\hline
\end{tabular}

$\dagger$ There was only one patient in stage 6 of liver fibrosis.

* $P$ value $\leq 0.05$

$* * \mathrm{P}$ value $\leq 0.001$

Table 4. Serum concentrations $(\mathrm{ng} / \mathrm{ml})$ of $\mathrm{LN}$ and PIIINP in various stages of liver fibrosis. Data are presented as Mean $\pm \mathrm{SD}(\mathrm{ng} / \mathrm{ml})$.

A cut-off point of $52.0 \mathrm{ng} \mathrm{LN} / \mathrm{ml}$ and $5.0 \mathrm{ng}$ PIIINP/ml for the discrimination of patients with liver fibrosis from those without liver fibrosis showed a good accuracy, AUC, sensitivity, specificity, PPV and NPV (Table 5). 


\begin{tabular}{|c|c|c|c|c|c|c|c|}
\hline Marker & AUC & P & Sen & Sp & PPV & NPV & Accu \\
\hline AAR & 0.907 & 0.001 & 86 & 76 & 77 & 69 & 85 \\
\hline AP & 0.632 & 0.179 & 68 & 55 & 64 & 45 & 59 \\
\hline APRI & 0.818 & 0.001 & 79 & 82 & 78 & 69 & 79 \\
\hline FIB4 & 0.754 & 0.001 & 77 & 81 & 76 & 65 & 76 \\
\hline FibroQ & 0.957 & 0.001 & 89 & 90 & 85 & 79 & 87 \\
\hline LN & 0.974 & 0.001 & 94 & 78 & 91 & 86 & 89 \\
\hline PIIINP & 0.873 & 0.001 & 85 & 70 & 89 & 60 & 81 \\
\hline
\end{tabular}

(AUC, area under the ROC curve; Sen, sensitivity; Sp, specificity; PPV, positive predictive value ;NPV, negative predictive value; Accu, accuracy).

Table 5. Accuracy of tests for discrimination of patients with liver fibrosis vs. control.

We selected other cut-off points (92.5 ng LN/ml and $8.9 \mathrm{ng}$ PIIINP/ml) for the discrimination of patients with mild fibrosis (stage 0-2) from severe fibrosis (stage 3-6) and the results are presented in Table 6. For comparison of the AUC of the serum marker panels (i.e. AP, AAR, APRI, FIB4 and FiroQ) with the AUC of the serum HA and LN levels, the AUC of these parameters were also calculated and the results are presented in table 5 and 6 , too.

\begin{tabular}{|c|c|c|c|c|c|c|c|}
\hline Marker & AUC & P & Sen & Sp & PPV & NPV & Accu \\
\hline AAR & 0.491 & 0.947 & 45 & 44 & 32 & 56 & 54 \\
\hline AP & 0.518 & 0.137 & 56 & 50 & 45 & 39 & 59 \\
\hline APRI & 0.268 & 0.107 & 29 & 22 & 31 & 28 & 33 \\
\hline FIB4 & 0.232 & 0.110 & 27 & 28 & 26 & 31 & 29 \\
\hline FibroQ & 0.491 & 0.997 & 44 & 45 & 33 & 55 & 56 \\
\hline LN & 0.879 & 0.005 & 86 & 71 & 87 & 69 & 82 \\
\hline PIIINP & 0.911 & 0.002 & 89 & 72 & 85 & 71 & 83 \\
\hline
\end{tabular}

(AUC, area under the ROC curve; Sen, sensitivity; Sp, specificity; PPV, positive predictive value ;NPV, negative predictive value; Accu, accuracy).

Table 5. Accuracy of tests for discrimination of patients with severe liver fibrosis vs. mild liver fibrosis.

Figures 3 and 4 are illustrated the results of ROC analysis of LN (a) and PIIINP (a) for discrimination of patients with liver fibrosis vs. control and also for discrimination of patients with severe liver fibrosis vs. mild liver fibrosis, respectively. 


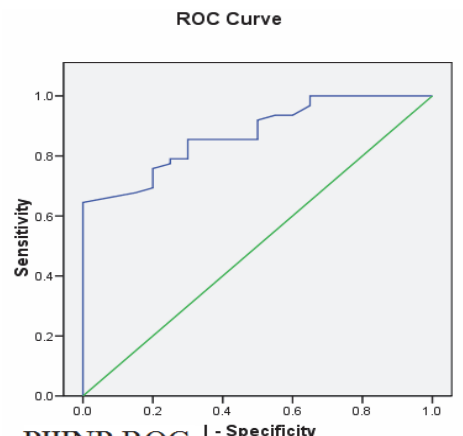

PIIINP ROC

a

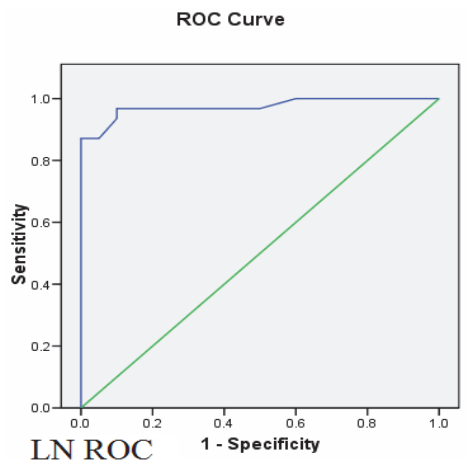

b

Fig. 3. ROC analysis of LN (a) and PIIINP (b) for discrimination of patients with liver fibrosis vs. control.

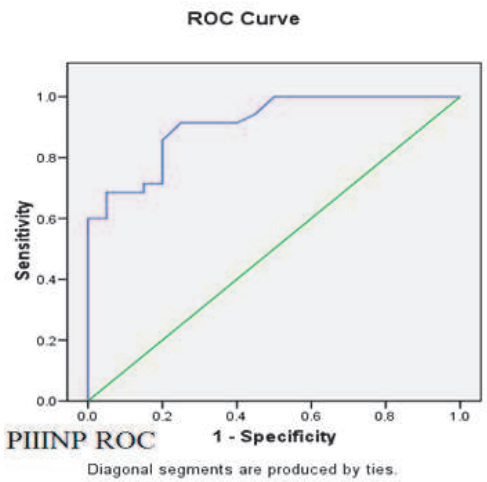

a

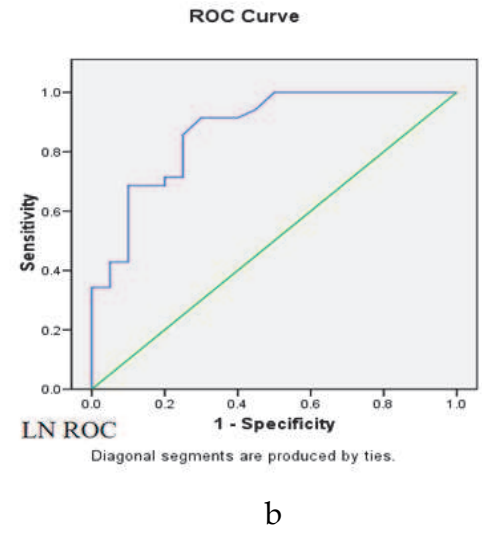

Fig. 4. ROC analysis of LN (a) and PIIINP (b) for discrimination of patients with severe liver fibrosis vs. mild liver fibrosis.

\section{Discussion}

Mean serum LN and PIIINP levels in chronic hepatitis B, C and auto immune hepatitis were significantly higher than in healthy controls $(\mathrm{p}<0.001)$. Patients with higher stages of liver fibrosis had higher serum LN and PIIINP concentrations and the correlations between serum LN and PIIINP concentrations with liver fibrosis stages were linear and strong (data not presented). Also, the mean serum LN and PIIINP concentrations in the stages 2, 3, 4 and 5 of the liver fibrosis showed statistically significant differences as compared with the healthy group. We didn't observe such a statistically differences in the stage 0 of liver fibrosis. In fibrosis Stage 6, which was represented by only one patient, we could not compare this single patient with the control group.

In addition, we observed that patients who had greater inflammation grades had higher serum LN and PIIINP concentrations (according to the figure 2) and the correlation of serum 
LN and PIIINP with the inflammation grades were linear (correlation data not presented). These findings suggest that patients with higher serum LN and PIIINP concentrations might have higher liver fibrosis stage and inflammation grade. Therefore we can conclude that when liver damage develops, the liver more poorly metabolized the LN and PIIINP. Patients in early stage of liver fibrosis possibly metabolized better LN and PIIINP in the liver than those with advanced liver fibrosis stages.

A cut-off point of $52.0 \mathrm{ng} \mathrm{LN} / \mathrm{ml}$ and $5.0 \mathrm{ng}$ PIIINP/ml for the discrimination of patients with liver fibrosis from those without liver fibrosis showed a good AUC (0.974 and 0.873, respectively), accuracy, sensitivity, specificity, PPV and NPV. Also serum marker panels in selected cut points (cut points not presented) showed reasonably good AUC (AP: 0.632, AAR: 0.907, APRI: 0.818, FIB4: 0.754 and Fibro Q: 0.957), accuracy, sensitivity, specificity, PPV and NPV. It means that all of these serum markers, individually or in a panel model, can discriminate between persons with liver fibrosis and healthy individuals.

A cut-off point of $92.5 \mathrm{ng} \mathrm{LN} / \mathrm{ml}$ and $8.9 \mathrm{ng}$ PIIINP/ml for discrimination of patients with mild fibrosis from those with severe fibrosis showed the AUCROC (p-value) of $0.879(0.005)$ and 0.911 (0.002), respectively. It means that LN and PIIINP can discriminate between patients with mild fibrosis from those with severe fibrosis. But the AUCROC (p-value) of serum panel markers for discrimination of severe liver fibrosis (stage $\geq 3$ ) than mild liver fibrosis (stage $\geq 2$ ) revealed a different pattern as follows: $A A R=0.491$ (0.947), $\mathrm{AP}=0.518$ (0.137), APRI $=0.268(0.107)$, FIB- $4=0.232(0.110)$ and Fibro $Q=0.491$ (0.997). It seems only the LN and PIIINP performed better at excluding advanced fibrosis than mild fibrosis.

The reported researches about these markers is controversial, but in recent years there is increasing evidences that show these markers can be used for assessing the liver fibrosis stages (Gressner et al.,2007). Serum level of LN, have been used by several authors as a noninvasive marker to assess liver fibrosis in patients with viral hepatitis. LN determination together with PIIINP, hydroxyproline, prothrombin activity, and AST/ALT ratio has been used in the diagnosis of advanced fibrosis in chronic hepatitis $C$ patients (Rosa \& Parise et al., 2008). Serum levels of PIIINP were studied in viral hepatitis patients and studies showed strong correlation between its levels and histological stage of hepatic fibrosis. Also PIIINP levels were in correlation with aminotransferase levels (Bensen et al., 1987).

Murawaki reported that differences in serum ALT and PIIINP levels between stage F0 (no fibrosis), F1 (mild fibrosis), F2 (moderate fibrosis) and F3 (severe fibrosis) in patients with chronic hepatitis $C$ were statistically significant (P-value: $<0.005,<0.0002$, respectively) (Murawaki et al., 2001). In a study by Attallah, mean level of PIIINP and LN in severe liver fibrosis stages were greater than mild liver fibrosis stages (Attallah et al., 2007). As well in additional reports, LN concentrations were increased in early stages of chronic liver disease and the highest concentrations were in active cirrhosis and chronic active hepatitis (Castera et al., 2000). 84).

The grater serum markers, in higher liver inflammation grades, reported by others (Parsian et al., 2009, Parsian et al., 2010). Zheng reported that serum fibrosis indices (PIIINP and LN) increased as the grade of inflammation aggravated (Zheng et al., 2002). Cai reported that LN was in correlation with liver histopathopathological injuries and inflammation grades (Cai et al., 2004). Also increased serum level of PIIINP and its association with the severity of liver necroinflammation in other studies reported (Murawaki et al., 1994).

Reported diagnostic criteria are not reliable and to some extent are controversial. There is some studies reported that PIIINP has reached a limited clinical application, but no 
widespread acceptance (Collazos \& Diaz, 1994). Sensitivities of about $76.0-78.0 \%$ and specificities of $71.0-81.0 \%$ have been reported, which can be increased up to $88.0 \%$, if combined with additional collagen fragment markers.

Zheng reported that in ROC curves analysis the AUC of PIIINP and LN was 0.800 and 0.463 , respectively. The analysis also showed that PIIINP have greater diagnosis performances than LN according to fibrosis staging (Zheng et al., 2002). Guechot reported that PIIINP and HA serum concentrations correlated with the histological grades of liver fibrosis in untreated patients with chronic viral hepatitis $C(P<0.001)$. In addition, ROC curves showed that serum HA had greater diagnostic performance than PIIINP both for discriminating patients with extensive liver fibrosis from those with no or mild fibrosis (AUC: 0.864 vs. 0.691, P <0.001) or for discriminating patients with cirrhosis from those without cirrhosis (AUC: 0.924 vs. 0.734, P <0.001) (Guechot et al., 1996).

There are various studies about the efficiency of serum marker panels. In a retrospective study, Snyder reported that APRI (with AUROC $=0.887$ ) is a simple biochemical index that has been shown to be useful and accurate in about $50 \%$ of patients with HCV (Snyder et al., 2007). Yilmaz reported that the APRI had an acceptable accuracy for the assessment of liver fibrosis in patients with chronic hepatitis $\mathrm{C}(\mathrm{CHC})$, but not in those with chronic hepatitis $\mathrm{B}$ (CHB) (Yilmaz et al., 2011). Wai evaluated the APRI in a cohort of 270 patients with CHC and reported that the AUCROC of the APRI for predicting significant fibrosis and cirrhosis were $0.80-0.88$ and $0.89-0.94$, respectively and concluded that the APRI can obviate liver biopsy in approximately $50 \%$ of patients (Wai et al., 2003).

In another study it has been shown that the AUCROC of the APRI for predicting significant fibrosis and cirrhosis were 0.76 and 0.82, respectively (Shaheen et al., 2008). The results of our study showed that APRI had sensitivity of $79.0 \%$ and specificity $82.0 \%$ for discrimination of patients with liver fibrosis vs. control and sensitivity of $29.0 \%$ and specificity $22.0 \%$ for discrimination of patients with severe liver fibrosis vs. mild liver fibrosis. In a study by Hsieh, the authors evaluated 140 patients with chronic viral hepatitis and calculated the APRI, AAR, and FibroQ for assessment of liver fibrosis (Hsieh et al., 2009). They concluded that FibroQ performed better than APRI, but was equal to AAR, in the prediction of significant fibrosis: [AUCROC (p-value): 0.783 vs $0.631(0.02)$ and 0.783 vs $0.733(\mathrm{p}=0.26)$, respectively] and cirrhosis [AUCROC ( $\mathrm{p}$-value): 0.791 vs 0.634 ( $\mathrm{p}=0.03$ ), and 0.791 vs 0.782 ( $p=0.47)$, respectively). Liu demonstrated a low accuracy of APRI and AAR for predicting significant fibrosis in viral hepatitis $C$ carriers with persistently normal ALT levels. The AUCROC in differentiating significant fibrosis were only 0.673 for APRI and 0.504 for AAR (Liu et al., 2006). In a study by Martinez, the performances of APRI and FIB-4 index were validated in 340 patients who underwent antiviral therapy (Martinez et al., 2011). APRI and FIB-4 showed comparable diagnostic accuracies for significant fibrosis (AUROC: 0.83 and 0.85, respectively). To identify cirrhosis, FIB-4 index showed a significantly better performance over APRI score (AUROC: 0.89 vs. 0.83 ).

In another study Zhang assessed the diagnostic value of FIB-4 in 212 chronic hepatitis B by comparing their results with histological features (Zhang et al., 2010). The AUCROC (pvalue) of FIB-4 for significant fibrosis, extensive fibrosis and cirrhosis were $0.733(<0.01)$, $0.746(<0.01), 0.756(<0.01)$, respectively. They concluded that the FIB-4 index is an accurate and inexpensive method to assess liver fibrosis in chronic hepatitis and may reduce the need for liver biopsy. Ladep compared the diagnostic validity of AP, APRI and AAR index to histology fibrosis stage among $90 \mathrm{CHB}$ patients (Ladep et al., 2007). The PPV of AAR, API 
and APRI scores were $52 \%, 63 \%$ and $54 \%$, with sensitivities of 60,11 and $96 \%$, respectively and interestingly the diagnostic accuracy of AAR for cirrhosis was $100 \%$. They concluded non-invasive fibrosis markers are not as sensitive for diagnosing significant fibrosis in chronic hepatitis $B$ compared to hepatitis $C$ patients and might have a limited utility for use in hepatitis B endemic populations.

Lackner evaluated diagnostic accuracies of AAR, AP and APRI index in 211 patients with chronic hepatitis $C$ and observed that diagnostic accuracy of APRI for prediction of significant fibrosis was superior to that of AAR and AP (Lackner et al., 2005). Fujii calculated AAR, AP, APRI indices in 100 patients with HCV. The AUC of the AAR, AP and APRI index for predicting cirrhosis were respectively $0.555,0.652$ and 0.761 . They concluded that these indices are good as noninvasive laboratory tests to predict cirrhosis in patients with HCV (Fujii et al., 2009).

Mahassadi conducted a prospective cohort study to determine the diagnostic accuracy of APRI, AAR, AP and FIB-4 index for the prediction of significant fibrosis or cirrhosis in 117 patients with CHB. APRI and FIB-4 index ruled out significant fibrosis with high specificity of $84.7 \%$ and $86.1 \%$, respectively and NPV of $78.2 \%$ and $72.9 \%$, respectively. More accurately, APRI or FIB-4 index ruled out cirrhosis with high sensitivity of $94.4 \%$ and $88.9 \%$ and high NPV of $98.1 \%$ and $96.3 \%$, respectively in another cut points. They suggested that APRI and FIB-4 index are simple readily available indices for assessment of liver fibrosis in CHB patients (Mahassadi et al., 2010).

Kim determined the clinical performances of AAR, AP and APRI index for the prediction of liver fibrosis in chronic viral liver diseases in 225 patients with chronic viral liver diseases (180 with hepatitis $B$ virus, 43 with hepatitis $C$ virus, and 2 with hepatitis $B+C$ virus) that underwent a liver biopsy procedure. The AUCROC for APRI, ARR were 0.822, 0.756, 0.642, respectively, for predicting significant fibrosis and were $0.691,0.711$ and 0.794 , respectively, for predicting extensive fibrosis. They concluded these serum markers were useful for predicting significant or extensive liver fibrosis in chronic viral liver diseases and APRI was most useful for the prediction of significant fibrosis (Kim et al., 2009).

As it is clear, numerous researchers have attempted to validate these findings, but the results were conflicting. Some researches as mentioned earlier, found a good AUCROC of these serum marker panels for discrimination between patients with mild fibrosis and those with severe fibrosis, but we didn't observe. The differences in patient populations, including the prevalence of significant fibrosis, differences in the reference ranges for AST, differences in the etiology and cause of liver fibrosis and also the pattern of the studies may in part explain these discrepancies (Yilmaz et al., 2011).

In summary, we observed that all serum markers including AAR, AP, APRI, FIB-4, FibroQ, LN, and PIIINP were useful for predicting liver fibrosis in chronic hepatitis patients compared with the healthy control, But we didn't observe such a similar pattern for discrimination between patients with mild fibrosis and those with severe fibrosis and only LN and PIIINP performed better at excluding advanced fibrosis than mild fibrosis and patients with liver fibrosis than healthy individuals. Therefore serum LN and PIINP measurement together with these tests could be used for predicting of liver fibrosis as alternate to liver biopsy, when liver biopsy is damaging.

\section{Conclusions}

Our findings suggest that measurement of serum LN and PIIINP concentrations can discriminate between patients with liver fibrosis and healthy individuals and between 
patients with mild fibrosis and those with severe fibrosis. An increase in serum LN and PIIINP concentrations above the predictive value is associated with liver fibrosis. In addition, the values of the AAR, AP, APRI, FIB-4 and FibroQ score and serum LN and PIIINP levels for discrimination between patients with liver fibrosis and healthy individuals are in the same order, but there is not such a similar pattern for discrimination between patients with mild fibrosis and those with severe fibrosis. It seems only the extracellular matrix components i.e. LN and PIIINP performed better at excluding advanced fibrosis than mild fibrosis and patients with liver fibrosis than healthy individuals. Therefore, serum LN and PIIINP levels together with the AAR, AP, APRI, FIB-4 and FibroQ score could be an additional non-invasive tool for evaluation of liver fibrosis, when liver biopsy is contraindicated. These indices may also be used for the assessment of therapeutic efficiency in chronic hepatitis patients, if liver biopsy cannot be performed.

\section{Acknowledgments}

The authors gratefully acknowledge the financial support from the Liver and Gastroenterology Research Centre, Tabriz University of Medical Sciences, Tabriz, Iran.

\section{References}

Attallah AM, Toson EA, Shiha GE, Omran MM, Abdelaziz MM, El-Dosoky. (2007). Evaluation of serum procollagen aminoterminal propeptide III, laminin, and hydroxyproline as predictors of severe fibrosis in patients with chronic hepatitis C.J Immunoassay Immunochem, 28, 199-211.

Bensen KD, Petersen K, Junker P. (1987). Serum aminoterminal procollagen type III in acute viral hepatitis. A long term follow-up study. Liver,7, 96-105.

Benyon D, Arthur MJP. (2001). Extracellular matrix degradation and the role of stellate cells. Semin Liver Dis, 21,373-384.

Bismut F, Ratziu V, Pieroni L, Charlotte F, Benhamou Y, Poynard T. (2001). Multivirc Group.Biochemical markers of liver fibrosis in patients with hepatitis $C$ virus infection: aprospective study. Lancet, 357, 1069-75.

Cai WM, Zhang BB, Weng HL, Hu ZR, Lv J, Zheng M, Liu RH. (2004). The diagnostic value of eight serum indices for liver fibrosis. Zhonghua Gan Zang Bing Za Zhi., 12,4, 219-22.

Castera L, Hartmann DJ, Chapel F, Guettier C, Mall F, Lons T, et al. (2000). Serum laminin and type IV collagen are accurate markers of histologically severe alcoholic hepatitis in patients with cirrhosis. J Hepatol, 32,3, 412-418.

Castera L, Pawlotsky JM. (2005). Noninvasive diagnosis of liver fibrosis in patients with chronic hepatitis C. MedGenMed. Nov 9,4, 39.

Collazos J, Diaz F. (1994). Role of the measurement of serum procollagen type III N-terminal peptide in the evaluation of liver diseases. Clin Chim Acta, 227, 37-43.

Crockett SD, Kaltenbach T, Keeffe EB. (2006). Do we still need a liver biopsy? Are the serum fibrosis tests ready for prime time? Clin Liver Dis. 10, 3, 513-34.

Fujii H, Enomoto M, Fukushima W, Ohfuji S, Mori M, Kobayashi S, Iwai S, Morikawa H,Tamori A, Sakaguchi H, et al. (2009).Noninvasive laboratory tests proposed for predicting cirrhosis in patients with chronic hepatitis $C$ are also useful in patients with non-alcoholic steatohepatitis. Journal of Gastroenterology,44, 6, 608-614. 
Granger DN, Kubes P. (1994). The microcirculation and inflammation modulation of leukocytre- endothelial cell adhesion. J Leukocyte Biol, 55, 662-675.

Gressner OA, Weiskirchen R, Gressner AM. (2007). Biomarkers of liver fibrosis: Clinical translation of molecular pathogenesis or based on liver-dependent malfunction tests. Clinica Chimica Acta, 381, 107-113.

Guechot J, Laudat A, Loria A, Serfaty L, Poupon R, Giboudeau J. (1996). Diagnostic accuracy of hyaluronan and type III procollagen amino terminal peptide serum assays as markers of liver fibrosis in chronic viral hepatitis $C$ evaluated by ROC curve analysis. Clin Chem, 42,558-63.

Hsieh YY, Tung SY, Lee IL, Lee K, Shen CH, Wei KL, Chang TC, Chuang CS, Wu CS, Lin YH. (2009). FibroQ: An Easy and Useful Noninvasive Test for Predicting Liver Fibrosis in Patients with Chronic Viral Hepatitis. Chang Gung Med J 32, 6, 614-622.

Ishak K, Baptista A, Bianchi L, Callea F, Groote J, Gudat F, et al. (1995). Histologic grading and staging of chronic hepatitis. J Hepatol, 22, 696-699.

Kao JH, Chen DS. (2002). Global control of hepatitis B virus infection. Lancet Infect Dis, 2, 7,,395-403.

Kim SM, Sohn JH, Kim TY, Roh YW, Eun CS, Jeon YC, Han DS, Oh YH. (2009). Comparison of various noninvasive serum markers of liver fibrosis in chronic viral liver disease. Korean J Hepato, 15(4):454-63.

Knodell RG, Ishak KG, Black WC, Chen TS, Craig R, Kaplowilz N, et al. (1981). Formulation and application of a numerical scoring system for assessing histological activity in symptomatic chronic active hepatitis. Hepatology, 1, 431 - 5.

Lackner C, Struber G, Liegl B, Leibl S, Ofner P, Bankuti C, Bauer B, Stauber RE. (2005).Comparison and Validation of Simple Noninvasive Tests for Prediction of Fibrosis in Chronic Hepatitis C. Hepatology, 41,6,1376-82.

Ladep NG, Okeke EN, Umar MS, Echejoh G, Malu AO. (2007). Comparison of Simple Non-Invasive Tests for Liver Fibrosis with Histology of Chronic Hepatitis B Patients of African Descent. Hungarian Medical Journal 4, 469-476.

Leroy V, Monier F, Bottari S. (2004). Circulating matrix metalloproteinases 1, 2, 9 and their inhibitors TIMP-1 and TIMP-2 as serum markers of liver fibrosis in patients with chronic hepatitis C: comparison with PIIINP and hyaluronic acid. Am J Gastroenterol, 99, 271-9.

Liu CH, Lin JW, Tsai FC, Yang PM, Lai MY, Chen JH, Kao JH, Chen DS. (2006). Noninvasive tests for the prediction of significant hepatic fibrosis in hepatitis $C$ virus carriers with persistently normal alanine aminotransferases. Liver Int,26, 1087-94.

Mahassadi AS, Attia AF, Bathaix FMY, Agbé1 NB, Doffou S, Kissi1 HY, Diomandé IM, Cales P, Ndri-Yoman T. (2010). Diagnostic accuracy of biochemical markers of fibrosis in black African patients with chronic hepatitis B. Health, 2, 1413-1420.

Manna MP, Strassburg CP. (2001). Autoimmune Hepatitis: Clinical Challenges. Gastroenterol, 120, 1502-1517.

Marcellin P, Asselah T, Boyer N. (2002). Fibrosis and Disease Progression in Hepatitis C. Hepatology, 36, S47- -56.

Marco V, Iacono O, Camma C, Vaccaro A, Giunta M, Martorana G, et al. (1999). The longterm course of chronic hepatitis B. Hepatology, 30, 257-64.

Marra F. (1999). Hepatic stellate cells and the regulation of liver inflammation. J Hepatol, 31, 1120-1130. 
Martinez SM, Fernández-Varo G, González P, Sampson E, Bruguera M, Navasa M, Jiménez W, Sánchez-Tapias JM, Forns X. (2011). Assessment of Liver Fibrosis before and after Antiviral Therapy by Different Serum Marker Panels in Patients with Chronic Hepatitis C. Alimentary Pharmacology \& Therapeutics, 33,1,138-148

Murawaki Y, Ikuta Y, Koda M, Kawasaki H. (1994). Serum type III procollagen peptide, type IV collagen 7S domain, central triplehelix of type IV collagen and tissue inhibitor of metalloproteinases in patients with chronic viral liver disease: relationship to liver histology. Hepatology, 20, 780-7.

Murawaki Y, Ikuta Y, Okamoto K, Koda M, Kawasaki H. (2001). Diagnostic value of serum markers of connective tissue turnover for predicting histological staging and grading in patients with chronic hepatitis C. J Gastroenterol, 36, 399-406

Naveau S, Poynard T, Benattar C, Bedossa P, Chaput JC. (1994). Alpha-2-macroglobulin and hepatic fibrosis. Diagnostic interest. Dig Dis Sci, 39, 2426-32.

Neighbors J. (2007). The Diagnosis and Management of Hepatitis C: The Role of the Physician Assistant. The Internet Journal of Academic Physician Assistants, 5, 2, 25-37.

Parsian H, Nouri M, Soumi MH, Rahimipour A, Qujeq D. (2009). Attenuation of serum laminin concentrations upon treatment of chronic hepatitis, N Z J Med Lab Sci, 63, 12-7.

Parsian H, Rahimipour A, Nouri M, Somi MH, Qujeq D, Fard MK, Agcheli K. (2010). Serum hyaluronic acid and laminin as biomarkers in liver fibrosis, J Gastrointestin Liver Dis, 19, 169-74.

Poynard T, Aubert A, Bedossa P, et al. (1991). A simple biological index for detection of alcoholic liver disease in drinkers. Gastroenterol, 100, 1397-402.

Poynard T, Bedossa P. (1997). Age and platelet count: a simple index for predicting the presence of histological lesions in patients with antibodies to hepatitis $C$ virus. METAVIR and CLINIVIR Cooperative Study Groups. J Viral Hepatol,4, 199-208

Regev A, Berho M, Jeffers LJ, et al. (2002). Sampling error and intraobserver variation in liver biopsy in patients with chronic HCV infection. Am J Gastroenterol, 97, 2614-8.

Rosa h, Parise ER. (2008). Is there a place for serum laminin determination in patients with liver disease and cancer? World J Gastroenterol, 21; 14, 3628-3632.

Rosenberg WM, Voelker M, Thiel R, et al. (2004). Serum markers detect the presence of liver fibrosis: a cohort study. Gastroenterol, 127, 1704-13.

Rossi E, Adams LA, Bulsara M, Jeffrey GP. (2007). Assessing Liver Fibrosis with Serum Marker Models. Clin Biochem Rev, 28, 1, 3-10.

Shaheen AA, Myers RP .(2008). Systematic review and meta-analysis of the diagnostic accuracy of fibrosis marker panels in patients with HIV/hepatitis C coinfection. HIV Clin Trials, 9,1,43-51.

Smedsr B, Perotoft H, Gustsfson S and Laurent B. (1990). Review article. Scavenger functions of the liver endothelial cell. Biochem J, 266, 313-327.

Snyder N, Nguyen A, Gajula L, Soloway R, Xiao SY, Lau DT, Petersen J. (2007). The APRI may be enhanced by the use of the FIBROSpect II in the estimation of fibrosis in chronic hepatitis C. Clin Chim Acta. ,381,2,119-23.

Stalnikowitz DK, Weissbrod AB. (2003). Liver fibrosis and inflammation. A review. Annals of Hepatology, 2, 4, 159-163. 
Sterling RK, Lissen E, Clumeck N, Sola R, Correa MC, Montaner J, et al. (2006). Development of a simple noninvasive index to predict significant fibrosis in patients with HIV/HCV coinfection. Hepatology, 43, 1317-1325.

Wai CT, Greenson JK, Fontana RJ, Kalbfleisch JD, Marrero JA, Conje evaram HS, et al. (2003). A simple noninvasive index can predict both significant fibrosis and cirrhosis in patients with chronic hepatitis C. Hepatology, 38,2, 518-26.

Wai CT, Greenson JK, Fontana RJ, Kalbfleisch JD, Marrero JA, Conjeevaram HS, et al.(2003). A simple noninvasive index can predict both significant fibrosis and cirrhosis in patients with chronic hepatitis C. Hepatology, 38, 2, 518-26.

Westin J, Lagging LM, Wejstal R, Norkrans G, Dhillon AP. (1999). Interobserver study of liver histopathology using the Ishak score in patients with chronic hepatitis $C$ virus infection. Liver, 19, 183-7.

Williams AL, Hoofnagle JH. (1988). Ratio of serum aspartate to alanine aminotransferase in chronic hepatitis Relationship to cirrhosis. Gastroenterology, 95, 734-9.

Yilmaz Y, Yonal O, Kurt R Bayrak M, Aktas B, Ozdogan O. (2011). Noninvasive assessment of liver fibrosis with the aspartate transaminase to platelet ratio index (APRI): Usefulness in patients with chronic liver disease. Hepat Mon, 11, 2, 103-107.

Zhang YF, Shi H, Chen LB, Xu QH. (2010). Value of FIB-4 for the diagnosis of liver fibrosis in chronic hepatitis B. Zhonghua Shi Yan He Lin Chuang Bing Du Xue Za Zhi, 24,3, 215-7.

Zheng M, Cai W, Weng H, Liu HR. (2002). ROC curves in evaluation of serum fibrosis indices for hepatic fibrosis. World J Gastroenterol, 8,6, 1073-1076.

Zweig MH, Campbell G. (1993). Receiver-operating characteristic (ROC) plots: a fundamental evaluation tool in clinical medicine. Clin Chem, 39, 4, 561-577. 


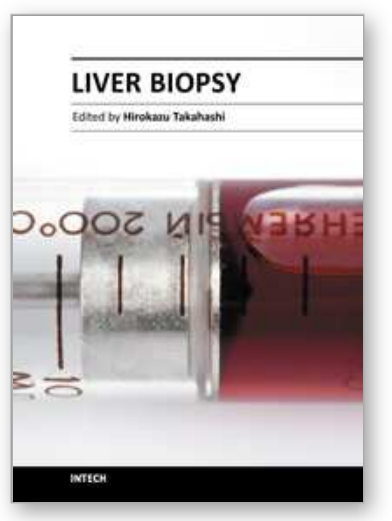

\author{
Liver Biopsy \\ Edited by Dr Hirokazu Takahashi
}

ISBN 978-953-307-644-7

Hard cover, 404 pages

Publisher InTech

Published online 06, September, 2011

Published in print edition September, 2011

Liver biopsy is recommended as the gold standard method to determine diagnosis, fibrosis staging, prognosis and therapeutic indications in patients with chronic liver disease. However, liver biopsy is an invasive procedure with a risk of complications which can be serious. This book provides the management of the complications in liver biopsy. Additionally, this book provides also the references for the new technology of liver biopsy including the non-invasive elastography, imaging methods and blood panels which could be the alternatives to liver biopsy. The non-invasive methods, especially the elastography, which is the new procedure in hot topics, which were frequently reported in these years. In this book, the professionals of elastography show the mechanism, availability and how to use this technology in a clinical field of elastography. The comprehension of elastography could be a great help for better dealing and for understanding of liver biopsy.

\title{
How to reference
}

In order to correctly reference this scholarly work, feel free to copy and paste the following:

Hadi Parsian, Mohammad Nouri, Ali Rahimipour, Mohammad Hossein Somi and Durdi Qujeq (2011). Comparison of Five Liver Fibrosis Indexes with Serum Levels of Laminin and N Terminal Peptide of Procollagen Type III in Chronic Hepatitis Patients, Liver Biopsy, Dr Hirokazu Takahashi (Ed.), ISBN: 978-953307-644-7, InTech, Available from: http://www.intechopen.com/books/liver-biopsy/comparison-of-five-liverfibrosis-indexes-with-serum-levels-of-laminin-and-n-terminal-peptide-of-pro

\section{INTECH}

open science | open minds

\section{InTech Europe}

University Campus STeP Ri

Slavka Krautzeka 83/A

51000 Rijeka, Croatia

Phone: +385 (51) 770447

Fax: +385 (51) 686166

www.intechopen.com

\section{InTech China}

Unit 405, Office Block, Hotel Equatorial Shanghai

No.65, Yan An Road (West), Shanghai, 200040, China

中国上海市延安西路65号上海国际贵都大饭店办公楼405单元

Phone: +86-21-62489820

Fax: $+86-21-62489821$ 
(C) 2011 The Author(s). Licensee IntechOpen. This chapter is distributed under the terms of the Creative Commons Attribution-NonCommercialShareAlike-3.0 License, which permits use, distribution and reproduction for non-commercial purposes, provided the original is properly cited and derivative works building on this content are distributed under the same license. 